\title{
Research of Visual Layout System of Production Line based on Virtual Simulation Technology
}

\author{
Qi Huang, Lilan Liu \\ Shanghai Key Lab of Intelligent \\ Manufacturing and Robotics \\ Shanghai University \\ Shanghai, China \\ 294436757@qq.com, lancy@shu.edu.cn
}

\author{
Sen Wang, Wei Zhou \\ Solution business department \\ Shanghai Baosight Software co., Ltd \\ Shanghai, China \\ wangsen@baosight.com,180705@baosight.com
}

\begin{abstract}
Layout design of the production line is a difficult problem directly affecting the productivity. This paper researches on a rapid method of dynamic layout for the production line in manufacturing industry. Based on virtual simulation technology, a visualization system of production line layout is developed using virtual simulation software called Unreal Engine. The developed layout design system is applied for a steel production line. Moreover, the production process simulation function of layout design system is used to simulate the production process of this production line. The reasonableness of production line layout is verified so that it can be modified and optimized easily.
\end{abstract}

Keywords-virtual simulation; production line layout; 3D modeling; Unreal Engine

\section{INTRODUCTION}

With the rapid development of computer technology, the advantages on visual expression and 3D dynamic simulation of $3 \mathrm{D}$ simulation software is becoming increasingly significant.

Before constructing the practical production line, it is helpful to construct the 3D virtual model and simulate process flow dynamically on the hardware layout, process planning, tact and capacity, distribution path and control mode in the production line, providing a realistic and intuitive platform for overall planning and design, and showing the desired effect in a virtual environment. Thereby, managers can take a reasonable assessment of the innovation of the overall program, the rationality of design, the progressiveness of technology and the difficulty of realization to achieve the goal of saving costs and cutting down the development cycle [1].

Production line layout will directly affect the efficiency of the whole system. Many factors need to be considered in the design of the production lines layout, including the selection of processing equipment, logistics equipment and various auxiliary equipment according to the type, yield, processing technic and other system characteristics. Furthermore, leading products, making spatial arrangement on these devices are also to be considered combining with the structural characteristics of workshop to ensure the automation of the overall system.

Most researches on production line layout problem are for logistics, enhancing the efficiency of logistics from the

Supported by National Science and Technology support program, No. 2015BAF22B01; Shanghai Economic and Information Technology Commission-funded project, No. 14511108303. perspective of making a flexible layout [2]. Layout process is mainly based on the constraints by some algorithm to optimize the production line, while the influence of subjective factors on the layout is ignored. The main purpose of this study is to build a visual design platform for production line layout based on the virtual simulation technology [3]. With the powerful interactive features of virtual simulation software, users can operate intuitively and design in real-time environment. Designer's experience can be integrated at the beginning of layout design, improving the superiority of the production line effectively [4].

\section{SYSTEM ARCHITECTURE}

\section{A. Design Process of Production Line Layout}

This paper takes advantages of currently popular virtual reality software in aspect of real-time interaction, and develops a man-machine interactive and visual system for production line layout design. The general idea is based on the process sequence of production line process and making arrangements for the place order of related production equipment. Generally, it can be divided into three steps: general division, detailed layout and construction with installation [5]. General division is to confirm the shape and the relationship between the individual operating units; detailed layout is to arrange each individual operating units or equipment in the workshop to determine its location.

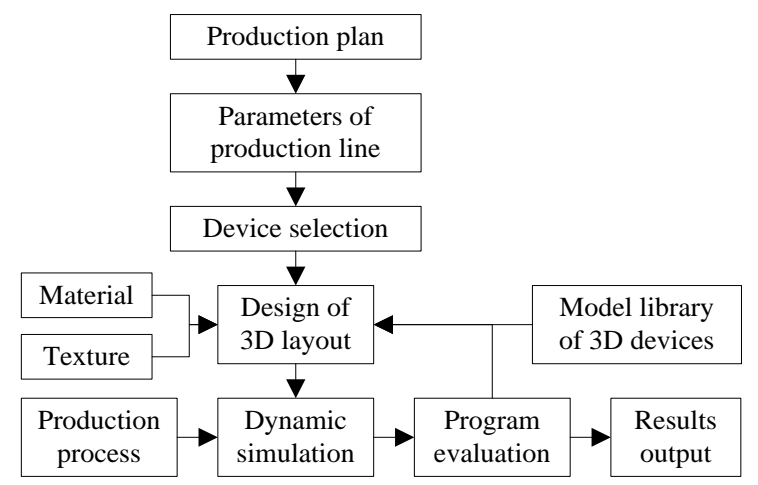

Fig. 1. Design process of production line layout

Production technology and production plan are regarded as the foundation of production line layout. Accordingly, virtual 
reality software is used to simulate the production process, modify and optimize the previous layout based on simulation results. Finally, results are exported as a guide of practical layout. Fig. 1 is a design flowchart of the layout design system.

\section{B. Framework of Layout Design System}

According to the chosen software development platform, the development of the layout design system includes two aspects:

\section{1) User interface}

User interface provides the interaction channel of layout design system for the production line layout designers. Under intuitive user interface, designers can manipulate with device models and scenes in real time. Designers can simulate the production process, and test the feasibility of layout programs. In this project, UMG module of Unreal Engine is used to build software window interface. The modular design is based on event-driven. UMG module provides rich widgets for the interface design, which can respond to multiple events. When the program runs, widgets are supposed to recognize corresponding events and then execute related codes.

\section{2) Back-stage functions}

Back-stage functions provide functional support for the production line layout design system, it is the backstage implementation of various operations in the user interface. There are the following three aspects:

- Basic functions: Basic functions include the basic functions of the application form class, including new, open, save, close, etc. With these functions, users can set the location, direction and magnitude of device models in the workspace, together with the movement, rotation and scaling of $3 \mathrm{D}$ view of workspace.

- Simulation function: When all the equipment is properly connected, the initial layout production line is completed. Users can make effect configuration through 3D animation. The software can simulate the process from material to finished production.

- Roaming function: Roaming is the core of interactivity of virtual tour system and the important interface between the user and a virtual scene. Users can intuitively understand the shortcomings of the production line and adjust in time combining the panoramic view with text description.

A complete back-stage function to support software can be built by combining the following four parts, creating a new file, establishing 3D device model, making the support program of operations and objects, and utilizing the database to build production line equipment model library for device management

\section{KEY TECHNOLOGIES OF SOFTWARE IMPLEMENTATION}

\section{A. 3D Modeling Techniques}

Equipment modeling is the basic segment of production line layout, its essence lies in how to express the relationship between the internal and external of production line equipment in the computer effectively.

The selected virtual reality software Unreal Engine itself does not have the modeling capabilities, equipment models that production line simulation needed should be achieved through external modeling software. In this paper, 3ds MAX is used, which has been widely applied in industrial product design, digital technology, simulation systems development and many other fields [6].

\section{B. Textures and Materials of Models}

In the building process of production line models, necessary materials will increase the texture and sense of reality of models. Texture mapping technology is an important technology widely used in computer graphics. For example, in the building process of steel production line, some models need to show in the form of textures, for instance, exterior color of devices, the wall and interior floor of workshop, etc. Fig. 2 shows the gravel texture model of slab yard inside factory building. Streamlining the data of model can be achieved by texture, making full use of design aesthetics will corporate culture and brand awareness into the build process of the production line, and build the integration of science and technology and cultural from the initial planning stage. Fig. 3 shows a roughing mill machine made by SMS Group.

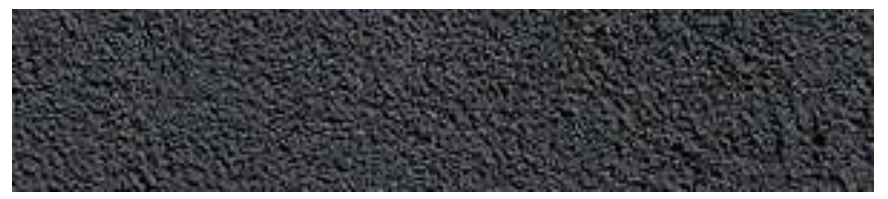

Fig. 2. Gravel texture of slab yard

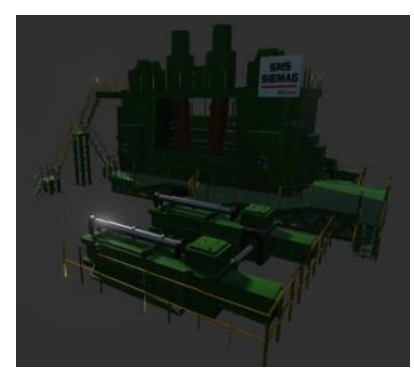

Fig. 3. Mapping model of SMS Group roughing mill machine

\section{Virtual Simulation Technology}

Virtual simulation technology is the combination of simulation technology and virtual reality technology. It takes building a uniform system for the unite and complete virtual environment as typical characteristics, and integrates and controls a large number of entities by virtual environments. Unreal Engine is a virtual reality software with powerful interactive features, it can easily realize the operation on models and the scene in real time interaction by mouse, keyboard and other interactive devices. Through directly calling internal BB (Building Block) of behavior module library to realize the control over models and scenes. Unreal Engine itself provides thousands of calls for BB, each BB encapsulates all the behavior of the control function and provides input-output interface parameters. Selecting suitable combination of BB can achieve a variety of operations on interactive 3D scenes and objects, such as movement, rotating, scaling, etc. Besides, Unreal Engine also provides a more powerful and flexible built-in visual programming module BP (Blue Print) and other tools for users to develop their own $\mathrm{BB}$ for some special and complex functions 
[7]. Unreal Engine integrates Visual Studio compiler, users can easily edit and run corresponding codes in real time to improve the efficiency of the script file. On some simple functional requirements, application of $\mathrm{BP}$ can quickly implement and test new ideas without creating a new $\mathrm{C}++$ program. Furthermore, since the BP embedded in the Unreal Engine, it can directly access the Unreal Engine development scenarios, and provides a feature-rich classes and functions, it can greatly simplify the operation and can achieve a more complex $3 \mathrm{D}$ virtual scene with dynamic interaction.

Fig. 4 and fig. 5 is a usage of Unreal Engine in BP visual programming module for 3D scene perspective operation. Fig. 4 is an implement of the zoom function of the perspective, you can zoom in and out the perspective by sliding the mouse wheel; Fig. 5 is an implement of the function of moving the scene perspective, you can move the viewing angle in any direction by holding the middle mouse button.

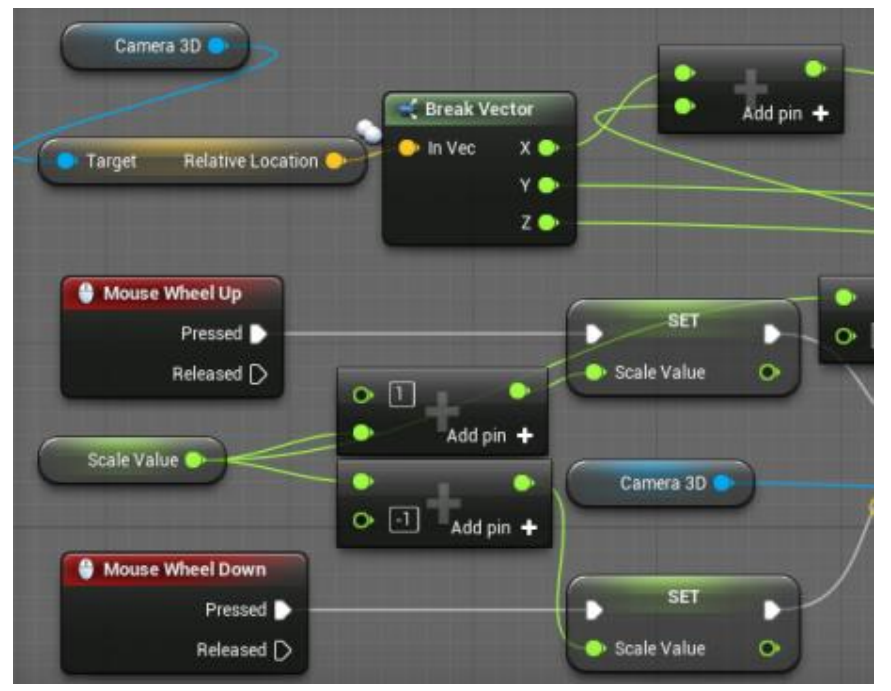

Fig. 4. Zoom function of scene perspective

Visualization system requires the use of the mouse to control the viewing angle, and use the W, A, S and D keys to control the movement of any direction. Open Unreal Engine and set the project, the configuration is shown in Fig. 6.

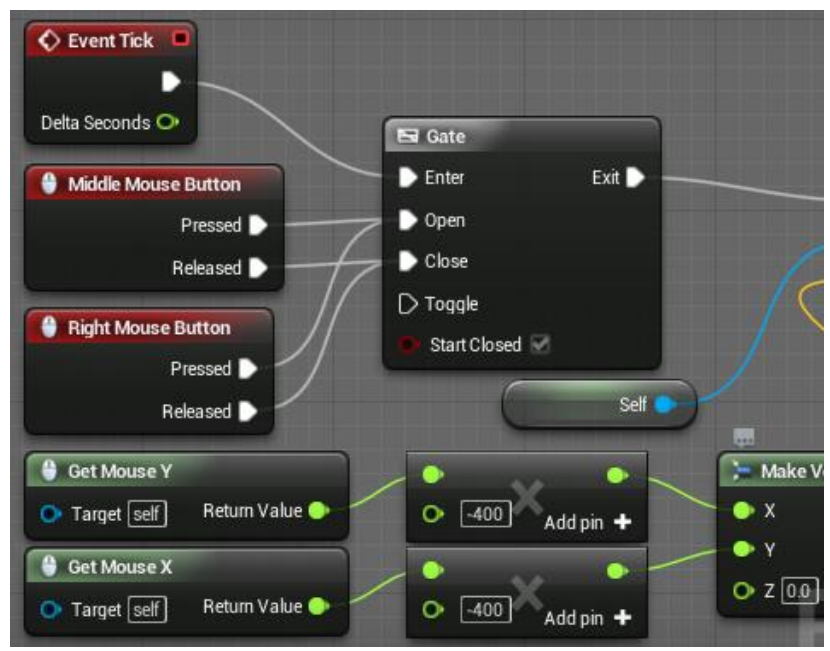

Fig. 5. Movement of scene perspective

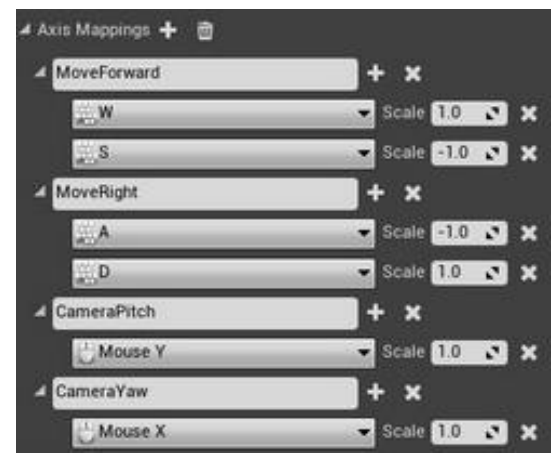

Fig. 6. Configuration of roaming control

In order to respond to the configuration, directly using the mouse to rotate the camera in the X-axis deflection, adding the following code to achieve the camera's deflection.

//Rotating actor deflection, this will rotate the camera, because the camera is attached to the actor

\section{\{}

FRotatorNewRotation=GetActorRotation();

NewRotation. Yaw+=CameraInput. X;

SetActorRotation(NewRotation);

\}

if(!MovementInput. IsZero())

\{ units

//Scaling the value of movement which input axis per 100

MovementInput=MovementInput. SafeNormal()*100. 0f;

FVectorNewLocation=GetActorLocation();

NewLocation+=GetActorForwardVector()*MovementInput. $\mathrm{X}^{*}$ DeltaTime;

NewLocation+=GetActorRightVector()*MovementInput. Y*DeltaTime;

SetActorLocation(NewLocation);

\}

\section{Equipment Model Library}

There are many types and large numbers of equipment in production line, in order to manage and easily to use, according to the current situation, collecting various types of equipment in the presence of production line, establishing equipment model library, and using Microsoft ACCESS database to manage it. All equipment on the production line are numbered, add parameters of the device to the data table. According to the given user equipment parameters, the system calls the corresponding equipment record, users can view the current equipment information and edit the information. With the equipment model library, users can easily take all equipment on the production line for unified management, and access to remove old equipment and add new equipment. 


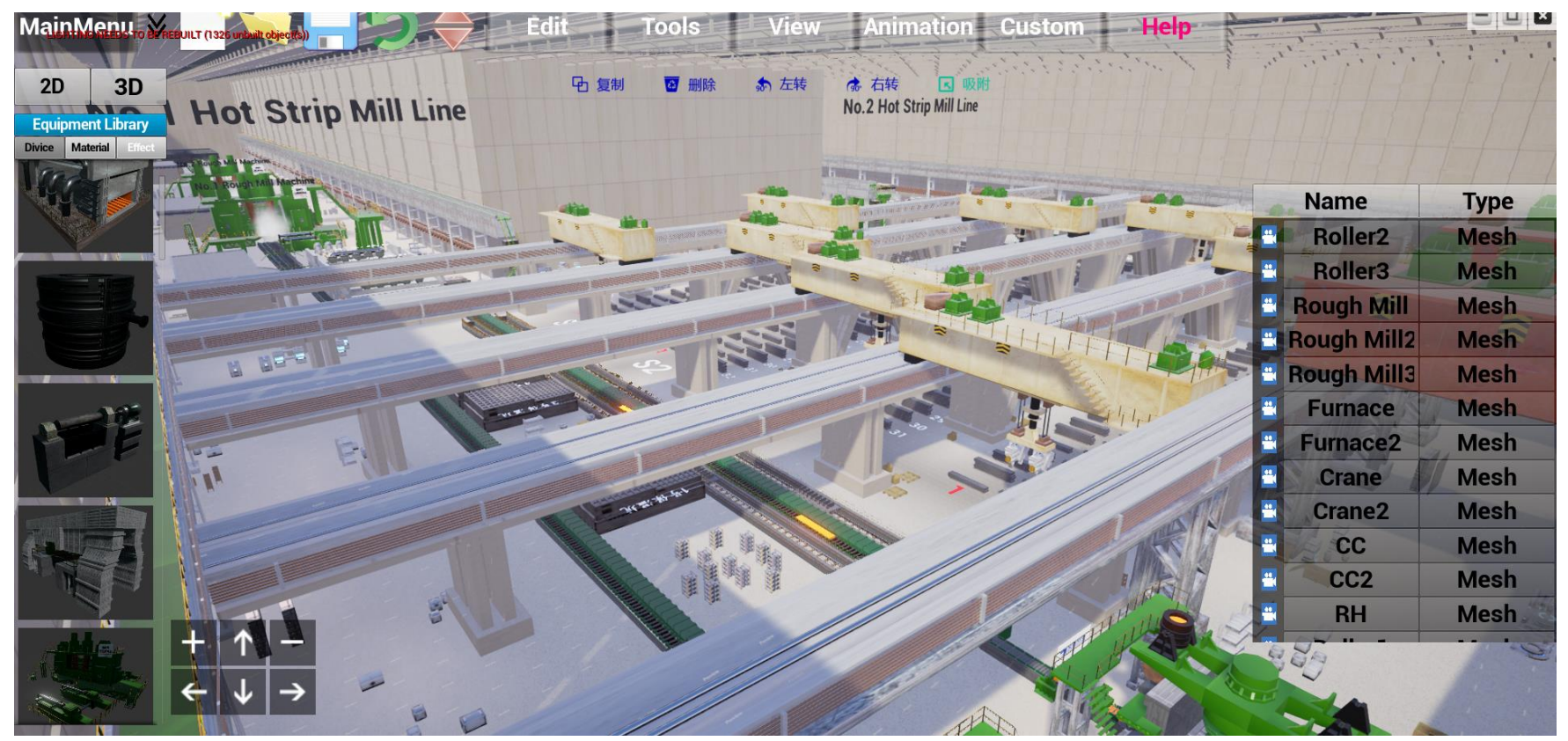

Fig. 7. A preliminary design of a steel production line layout

\section{A SteEl Production Line LAyout DESIGN EXAMPLES}

With this production line layout design system, taking a new steel production line of an iron and steel enterprise for example. Here is the specific design process: left-click the device tree node on the left of the interface, select the device into a 3D scene, then select operational button on the toolbar or right-click menu item to move, rotate and scale the equipment and locate the equipment in the scene, after that use the same method to import all the equipment needed, add rollers or pipes and other connected devices depending on the type of material transfer between devices, so the devices are connected together to form a complete production line. As it shown in Fig. 7. Finally, based on the actual production process and production methods to simulate the production process of the production line, according to the simulation results, make changes and optimization to the layout.

\section{CONCLUSION}

Production line simulation design and planning realizes the integration of advanced design concepts, modes of production and manufacturing technology under virtual environment [8].

With continuous development information technology and computer hardware and software technology in the manufacturing industry, further integration of virtual manufacturing and actual production, huge improvement of rapid modeling, process simulation and optimization algorithms, virtual simulation technology in the design and planning of advanced production lines will usher in a new stage of development.

The research work is currently still in its infancy, and it's mainly based on the designer's experience to design the layout, excellent layout still depends on the extent of the degree of experienced designers, there are still many problems with the accuracy and the degree of optimization, so the combination of usage of constraint and optimization method will be considered to optimize the system later. Moreover, the function of simulation software Unreal Engine used in this paper is very strong, we will continue to study and make deeper development, such as the logistics system simulation, optimization based on feedback on the production line layout, etc. On the basis of indepth study, more useful functions will be developed and be optimized.

\section{ACKNOWLEDGMENT}

The authors would like to express appreciations to colleagues and mentors in Shanghai Baosight software corporation and Shanghai University for their valuable comments and other helps.

\section{REFERENCES}

[1] Wang H. F. and Wan L., "Construction of production line planning of aircraft CNC machining based on virtual simulation technology," Aeronautical Manufacturing Technology. 493(23/24): 71-74, 2015.

[2] Li H. L. and Chen L., "Application of visual simulation to workshop system planning," Computer Systems \& Applications, (5): 162-165, 2012.

[3] Zhou J., Liu Z. Q., Deng J. X. and Ai X., "Virtual design of production line and its simulation application," Manufacturing Technology \& Machine, 2005 (1): 13-18.

[4] Liu L. J., "Visualization application of virtual reality in engineering," Mechanical Design, 29(1): 1-4, 2012.

[5] Xu L., Jiang Z. and Lu H., "The application of virtual reality technology in constructing virtual machining workshop," Intelligent Robotics and Applications. Springer Berlin Heidelberg, 2008: 479-487.

[6] Wang X. B. and Zhu W. J., "Three dimensional modeling based on OpenGL and 3ds MAX," Electronic Science and Technology, 25(1): 7980, 2012.

[7] Ryan S, Mastering the art of unreal engine 4 - blueprints, CreateSpace Independent Publishing Platform, 2014.

[8] Sun L. S., Ning R. X. and Wang X. Y., "Visual design of production line in virtual manufacturing," Journal of Beijing Institute of Technology, 22(1): 32-35, 2002. 